\title{
Estimating sensory texture of cooked rice using full and optimized predictive regression models
}

\author{
Youngseung Lee ${ }^{1 \dagger}$, Han Sub Kwak ${ }^{1,2 \dagger}$, Marura Lenjo ${ }^{3}$, Jean François Meullenet ${ }^{3 *}$ \\ ${ }^{1}$ Department of Food Science and Nutrition, Dankook University, Yongin-si 448-701, Korea; ${ }^{2}$ Natural Nutraceuticals Industrialization Research \\ Center, Dankook University, Cheonan-si 330-714 Korea; ${ }^{3}$ Department of Food Science, University of Arkansas, Fayetteville, AR 72701 USA; \\ tThese authors contributed equally to this study
}

\section{A B S TR A C T}

\begin{abstract}
The objective of this study was to predict descriptive texture characteristics of cooked rice with a texture analyzer using a full predictive model (partial least square regression; PLSR) and an optimized predictive model (jackknife resampling method; JRM). Texture profiles of 102 cooked rice samples were measured using a spectral stress strain analysis. Eleven sensory texture characteristics were evaluated using a trained descriptive panel. The JRM showed slightly better prediction for sensory texture attributes than PLSR due to the removal of insignificant variables. The following three sensory attributes were strongly predicted by JRM based on the calibration model correlation coefficient (Rcal): cohesion of bolus (Rcal $=0.78$ ), adhesion to lips (Rcal $=0.83$ ), and hardness (Rcal $=0.72$ ). Cohesiveness, toothpull and toothpack were moderately predicted (Rcal $\geq 0.60$ ). The results from the texture analyzer were able to estimate sensory texture attributes, which were directly related to texture characteristics such as hardness, stickiness and cohesiveness.
\end{abstract}

Keywords: Rice; Texture; Partial least square regression; Jackknife resampling

\section{INTRODUCTION}

Estimating food quality by correlating sensory evaluation results and instrumental measurement has been widely studied (Chen, et al., 2005; Jeong, et al., 2013; Lee, et al., 2014). Texture characteristics of cooked rice play a major role in the selection of properly cooked rice (Nishinari, 1996). Recently, estimating cooked rice quality by instrumental measurements - Toyo taste meter and eating quality indicator - has been conducted by correlating consumer acceptance ratings in order to find out which instrumental parameters were related with consumer acceptance. Kwak et al. (2015a) have shown differences in drivers' liking and disliking of aseptic-packaged cooked rice based on consumer acceptance and two instrumental measurements using partial least square regression (PLSR) analysis. The instrumental measurements were not highly correlated with consumer acceptability or many descriptive attributes in aseptic-packaged cooked rice. For frozen-cooked rice, hardness and stickiness based on the texture analyzer were highly correlated with consumer acceptance (Kwak et al., 2015b). However, these studies were mainly focused on the relationship between consumer acceptance and instrumental quality measurement rather than the relationship between attribute intensities and texture profiles.

Several researchers have studied instrumental evaluation of cooked rice texture in order to estimate the intensities of texture attributes from descriptive analyses. One of the widely used instrumental texture measurement methods was Ottawa extrusion cell (Juliano, et al., 1984; Meullenet, et al., 1998). However, the dimensions of traditional Ottawa cell require a large amount of samples (approximately $100 \mathrm{~g}$ ) for evaluation. After the introduction of Ottawa cell for rice texture measurement, a extrusion cell method with lower amount of cooked rice was designed to measure cooked rice texture (Sitakalin and Meullenet, 2000). The results showed a more accurate predictive model by the small extrusion cell analysis. Applying appropriate statistical methods to analyze these results are necessary in order to deliver a better estimation model. Traditionally PLSR was used for the estimation by using the entire results (Kwak et al., 2015a; Meullenet, et al., 1998). If there are noise results that distort the average results, these outliers would be negatively influence the validation of the estimation model. Jackknife resampling method (JRM) is the statistical method for optimization by deleting noise data

\footnotetext{
*Corresponding Author: Jean François Meullenet, Department of Food Science, University of Arkansas, 2650 N. Young Avenue, Fayetteville, AR 72704. Phone: (479) 575-6822, Fax: (479) 575-6936, E-mail: jfmeull@uark.edu.

Received: 28 September 2015; Revised: 22 November 2015; Accepted: 24 November 2015; Published Online: 24 November 2015
} 
(higher than \pm 2 standard deviations) from the full statistical model (Martens and Martens, 2001). By removing the noise data, there would be a higher chance to generate a more robust predictive model for estimation. Therefore, the objective of this study was to estimate sensory texture attributes of cooked rice by an instrumental texture measurement named spectral stress strain analysis (SSSA) using two regression models, PLSR analysis and JRM.

\section{MATERIALS AND METHODS}

\section{Rice samples}

All varieties were harvested from the University of Arkansas Rice Research and Extension Center at Stuttgart, AR, USA. Moisture contents of harvested rice were ranged $17-19 \%$ (wet base). Three long grain rice varieties (Oriza sativa var. Drwe, Bengal, and Kaybonnet) were used for the evaluation and total numbers of rice samples were 102 . Samples were dried $(12-13 \% \mathrm{w} / \mathrm{w})$ and stored by the University of Arkansas Rice Processing Plant.

\section{Descriptive analysis}

Eleven trained panelists with three years of experience in Spectrum descriptive analysis method (Meilgaard, et al., 1999) evaluated eleven texture attributes for 102 cooked rice samples. Attributes evaluated and their definitions are presented in Table 1. The eleven attributes were intensified during four evaluation stages following the general procedure for eating cooked rice. During the initial stage of evaluating cohesion of bolus, particles size and adhesiveness to lips were evaluated. In the second stage, hardness and cohesiveness were evaluated by compressing samples between lips followed by releasing the samples to determine the intensities of attributes. During the chewdown stage that panelists used to masticate the cooked rice, cohesiveness of mass, roughness of mass, and toothpull were evaluated. During the last stage, the panelists evaluated toothpack, loose particles, and residual film related to mouthfeel after eating cooked rice.

Rice (300 g) was washed using a tap water and then put into an electronic rice cookers (National, model SR-W10FN, National Co., Osaka, Japan) with $600 \mathrm{~g}$ of tap water (Meullenet, et al., 1999). Samples were transferred to glass bowls preheated at $75 \pm 2^{\circ} \mathrm{C}$, and the glass bowls were put into Styrofoam cups and covered with watch glasses to minimize temperature change. Water and soda cracker were provided to individual panelists to clean their palate between samples. The panelists evaluated one sample at a time in individual booths under incandescent lighting. During the evaluation sessions, fifteen samples were presented at each session and twelve samples were presented at the last session with a random sample order. A warm-up sample was presented to the panelists prior to the actual evaluations. Panelists used paper ballots and the 16-point category scale labelled from ' 0 ' on the left side and ' 15 ' on the right side.

\section{Instrumental texture analysis}

Temperature was closely monitored during the test because it could affect rice texture (Okabe, 1979). Room temperature was not effective for measuring texture of cooked rice (Meullenet, et al., 1998; Meullenet, et al., 1999). In addition, room temperature could be different from the temperature of cooked rice for descriptive analysis. In order to maintain temperature of the cooked rice same as the descriptive analysis, individual small batch cooking was applied. Briefly, milled rice (10.0 g) and water (17 g) were put into a $100 \mathrm{~mL}$ beaker and cooked using an electronic rice cooker (National, model SR-W10FN) under steam conditions. For uniform cooking, beakers were placed at the center of the cooker and had $1 \mathrm{~cm}$ distance from the side of the cooker. Water $(350 \mathrm{~mL})$ was added to the ricecooker for equivalent heat transfer to the beakers. Rice was steamed for $30 \mathrm{~min}$ with the regular cooking option.

The measurement of cooked rice texture was performed using SSSA (Sitakalin and Meullenet, 2000). Extrusion cells were removed to be cleaned one at a time from the rice cooker. Instrumental testing was performed immediately after getting out of a beaker from the rice cooker. Instrumental texture measurement was conducted using a texture analyzer (model TA-XT2i, Texture Technologies, Scarsdale, NY, USA). The testing conditions were a $25 \mathrm{Kg}$ load cell, $2.0 \mathrm{~mm} / \mathrm{s}$ of cross-head speed, and $85 \mathrm{~mm}$ total distance. A typical force deformation curve and stages by SSSA is shown in Fig. 1. The curve was divided into four stages; packing, compression, extrusion, and tension (Fig. 1).

\section{Statistical analysis}

Unscrambler (version 7.5, CAMO, Throndheim, Norway) was used to generate predictive models in this study. PLSR was used for predicting descriptive sensory attributes from force-distance data of the texture profile analysis. Full cross-validation method was employed to evaluate model robustness. The JRM was employed to remove instrumental variables that created noise in the evaluations. The calibration model correlation coefficient (Rcal), full cross validation correlation coefficient (Rval), root mean square error of calibration (RMSEC), and root mean square error of prediction (RMSEP) and robustness (RMSEP/RMSEC) were presented to compare two predictive models (Lee, et al., 2008).

\section{RESULTS AND DISCUSSION}

Texture attributes of cooked rice by sensory evaluations were predicted using an instrumental measurement (Kwak, 
Table 1: Attribute, definition and tasting procedure for cooked rice

\begin{tabular}{|c|c|c|}
\hline Attribute & Definition & Tasting procedure \\
\hline \multicolumn{3}{|l|}{ Initial stage } \\
\hline Cohesion of bolus & $\begin{array}{l}\text { The degree to which the unchewed sample holds } \\
\text { or ticks together }\end{array}$ & $\begin{array}{l}\text { Place } 1 / 4 \text { teaspoon of sample in mouth and immediately } \\
\text { evaluate how tightly the mass is sticking or holding together. } \\
\text { Do not chew or manipulate! }\end{array}$ \\
\hline \multirow[t]{2}{*}{ Particle size } & $\begin{array}{l}\text { The amount of space the particle takes up in } \\
\text { the mouth }\end{array}$ & Place sample in center of mouth and evaluate \\
\hline & (How big are the particles?) & Do not chew or manipulate! \\
\hline Adhesion to lips & $\begin{array}{l}\text { The degree to which the sample adheres } \\
\text { to the lips }\end{array}$ & $\begin{array}{l}\text { Compress sample between lips, release and evaluate the } \\
\text { degree to which the product remains on the lips. }\end{array}$ \\
\hline \multicolumn{3}{|l|}{ First bite/chew } \\
\hline Hardness & The force required to compress the sample & $\begin{array}{l}\text { Compress or bite through sample one time with molars or } \\
\text { incisors }\end{array}$ \\
\hline Cohesiveness & $\begin{array}{l}\text { The amount the sample deforms rather than splits } \\
\text { apart, cracks or breaks }\end{array}$ & $\begin{array}{l}\text { Place sample between the molar teeth and compress fully. } \\
\text { May also be done with incisors }\end{array}$ \\
\hline \multicolumn{3}{|l|}{ Chewdown } \\
\hline Cohesiveness of mass & The amount that the chewed sample holds together & $\begin{array}{l}\text { Chew sample with molar teeth up to } 15 \text { times and evaluate } \\
\text { (Loose mass---Tight mass) }\end{array}$ \\
\hline \multirow[t]{2}{*}{ Roughness of mass } & $\begin{array}{l}\text { The amount of roughness perceived on the } \\
\text { surface of the chewed sample }\end{array}$ & $\begin{array}{l}\text { Chew the sample with molars and evaluate the irregularities } \\
\text { on the surface of the sample mass }\end{array}$ \\
\hline & $\begin{array}{l}\text { Hint: You are looking for the large lumps, bumps, } \\
\text { hills and valleys, etc. }\end{array}$ & \\
\hline Toothpull & $\begin{array}{l}\text { The force required to separate the jaws during } \\
\text { mastication }\end{array}$ & Chew sample 2-3 times and evaluate. \\
\hline \multicolumn{3}{|l|}{ Residual } \\
\hline Residual film & $\begin{array}{l}\text { The amount and degree of residue felt by the } \\
\text { tongue when moved over the surface of the mouth }\end{array}$ & $\begin{array}{l}\text { Swallow the sample and feel the surface of the mouth with the } \\
\text { tongue to evaluate }\end{array}$ \\
\hline Toothpack & $\begin{array}{l}\text { The amount of product packed into the crowns of } \\
\text { your teeth after mastication }\end{array}$ & $\begin{array}{l}\text { Chew sample } 10-15 \text { times, expectorate and feel the surface of } \\
\text { the crowns of the teeth to evaluate }\end{array}$ \\
\hline Loose particles & $\begin{array}{l}\text { The amount of particles remaining in and on the } \\
\text { surface of the mouth after swallowing }\end{array}$ & Chew sample with molars, swallow and evaluate \\
\hline
\end{tabular}

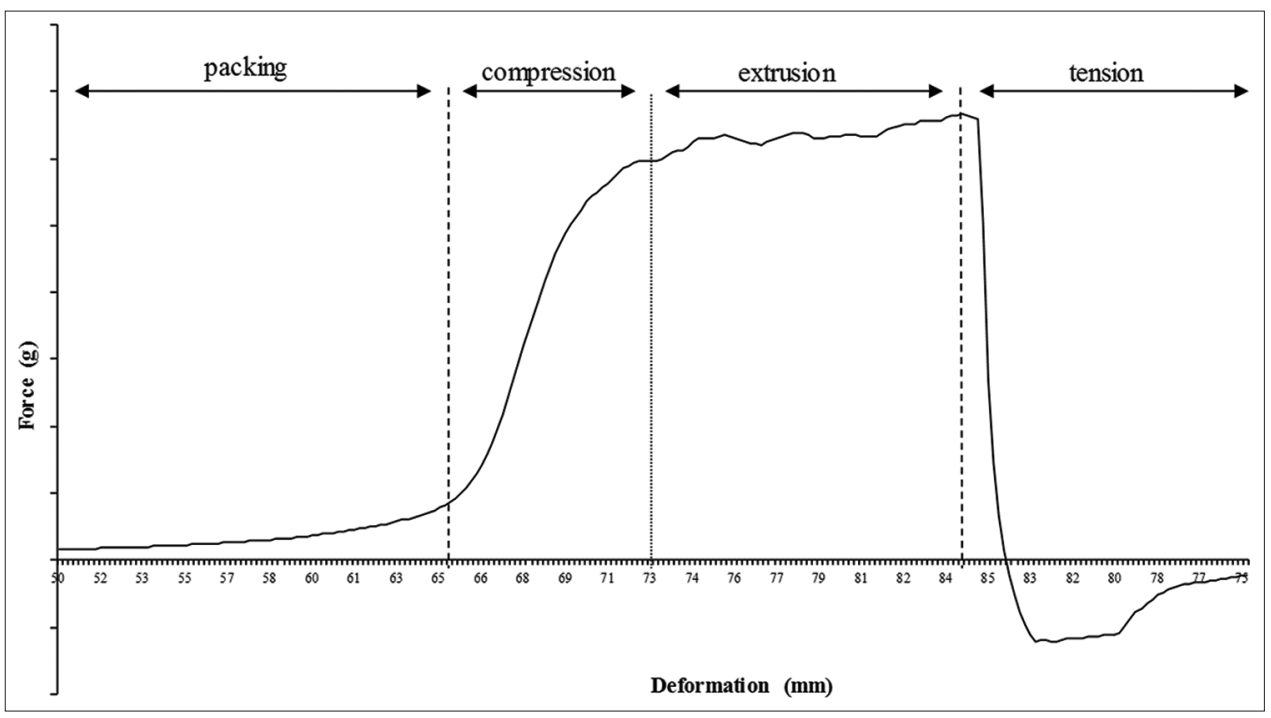

Fig 1. A typical force deformation curve of cooked rice by the spectral stress strain analysis (From Lenjo and Meullenet, 2000, with the written permission from the editor of Discovery Magazine, The Student Journal of Dale Bumpers College of Agricultural, Food and Life Sciences, University of Arkansas).

et al., 2015a; Meullenet, et al., 1999). Predictive parameters for PLSR analysis and JRM were shown in Table 2. The robustness values for each attribute were close to 1 in Table 2; therefore, the statistical models in this study were appropriate (Lee, et al., 2008). For the attributes in the initial stage, cohesion of bolus and adhesion to lips were well predicted by two statistical models. Cohesion of bolus $($ Rcal $=0.71)$ showed significant improvement of the model statistics by JRM (Rcal $=0.78)$. Especially, Rval (0.72) by the JRM was better than that (0.64) from 
Table 2: Full and optimized predictive model statistics of rice sensory texture attributes by partial least square regression (Part of the table from Lenjo and Meullenet, 2000, with the written permission from the editor of Discovery Magazine, The Student Journal of Dale Bumpers College of Agricultural, Food and Life Sciences, University of Arkansas)

\begin{tabular}{|c|c|c|c|c|c|c|c|c|c|c|}
\hline \multirow[t]{2}{*}{ Attribute } & \multicolumn{5}{|c|}{ Full predictive model } & \multicolumn{5}{|c|}{ Optimized predictive model } \\
\hline & Rcal $^{1}$ & RMSEC $^{2}$ & Rval $^{3}$ & RMSEP ${ }^{4}$ & Robustness $^{5}$ & Rcal $^{1}$ & RMSEC $^{2}$ & Rval $^{3}$ & RMSEP $^{4}$ & Robustness $^{5}$ \\
\hline \multicolumn{11}{|l|}{ Initial stage } \\
\hline Cohesion of bolus & 0.71 & 0.52 & 0.64 & 0.57 & 1.10 & 0.78 & 0.43 & 0.72 & 0.49 & 1.14 \\
\hline Particle size & 0.30 & 0.07 & 0.22 & 0.07 & 1.00 & 0.46 & 0.06 & 0.42 & 0.06 & 1.00 \\
\hline Adhesion to lips & 0.75 & 0.68 & 0.68 & 0.75 & 1.10 & 0.83 & 0.57 & 0.76 & 0.67 & 1.18 \\
\hline \multicolumn{11}{|l|}{ First bite/chew } \\
\hline Hardness & 0.69 & 0.28 & 0.58 & 0.32 & 1.14 & 0.72 & 0.27 & 0.69 & 0.28 & 1.04 \\
\hline Cohesiveness & 0.44 & 0.29 & 0.33 & 0.31 & 1.07 & 0.69 & 0.17 & 0.61 & 0.19 & 1.12 \\
\hline \multicolumn{11}{|l|}{ Chewdown } \\
\hline Cohesiveness of mass & 0.55 & 0.46 & 0.42 & 0.51 & 1.11 & 0.58 & 0.46 & 0.46 & 0.50 & 1.09 \\
\hline Roughness of mass & 0.62 & 0.26 & 0.53 & 0.28 & 1.08 & 0.51 & 0.28 & 0.47 & 0.29 & 1.04 \\
\hline Toothpull & 0.56 & 0.19 & 0.47 & 0.20 & 1.05 & 0.60 & 0.18 & 0.46 & 0.20 & 1.11 \\
\hline \multicolumn{11}{|l|}{ Residual } \\
\hline Residual film & 0.12 & 0.19 & -0.10 & 0.20 & 1.05 & 0.32 & 0.19 & 0.27 & 0.19 & 1.00 \\
\hline Toothpack & 0.59 & 0.22 & 0.47 & 0.25 & 1.14 & 0.60 & 0.22 & 0.44 & 0.25 & 1.14 \\
\hline Loose particles & 0.32 & 0.35 & 0.19 & 0.36 & 1.03 & 0.18 & 0.36 & 0.08 & 0.36 & 1.00 \\
\hline
\end{tabular}

${ }^{1}$ Rcal: Correlation coefficient of calibration model, ${ }^{2}$ RMSEC: Root mean square error of calibration, ${ }^{3}$ Rval: Full cross validation correlation coefficient, ${ }^{4} \mathrm{RMSEP}$ : Root mean square error of prediction, ${ }^{5}$ Robustness: RMSEP/RMSEC

the PLSR. The RMSEC (0.43) and RMSEP (0.49) of the optimized model were lower than those from the full predictive model. This meant that the data are located close to the average of the data (RMSEC) and the predictive model (RMSEP). Adhesion to lips was the best predictive attributes by the texture analyzer. Rcal by PLSR was 0.75 ; while the Rval by the PLRS was lower as of 0.68. The JRM showed lower RMSEC and RMSEP than those values from the PLSR analysis. JRM showed better prediction for adhesion to lips than PLSR. Contrary to the cohesion of bolus and adhesion to lips, particle size was poorly predicted by optimized model $($ Rcal $=$ 0.30). Optimization of the model resulted in a slight improvement in the correlation coefficient $(\mathrm{Rcal}=0.46)$. Particle size was not closely related to the texture of cooked rice but related to the shape so that there was a low correlation coefficient between the results from the descriptive analysis and the texture analyzer. Kwak et al. (2015a) reported low correlation coefficients between instrumental measurements and descriptive attributes of aseptic-packaged cooked rice for indirect attributes such as color, transparency, and kernel size, which were the appearance rather than the texture attributes.

For the first bite/chew stage, hardness was well predicted by the texture analyzer $(\mathrm{Rcal}=0.69)$ for the full model with a low RMSEC (RMSEC $=0.28$, Table 2). The optimized model had a correlation coefficient of Rcal $=0.72$ and RMSEC $=0.27$, which showed slight improvement compared to the full model. The optimized predictive model showed better prediction cohesiveness. The correlation coefficient for the full model for cohesiveness was 0.44 , while the optimized model had 0.69. Improvement of the model statistics was shown removing outliers of the results from the texture analyzer throughout the JRM. The RMSEC and RMSEP became lower as the JRM applied for cohesiveness.

In chewdown stage, the attributes were moderately estimated by presenting $0.51-0.62$ of Rcal. Cohesiveness of mass and toothpull were slightly better by the JRM when looking at Rcal and Rval (Table 2). Removing outliers were not well effective for these attributes. The full optimized model showed better prediction of roughness of mass $($ Rcal $=0.62)$ than the optimized model $(\mathrm{Rcal}=0.51)$. Since roughness of mass was the attributes for the perception of surface of cooked rice mass in mouth (Table 1), it was close to the surface feeling during masticating of the sample and not directly related to the texture characteristics measured by the texture analyzer.

For residual attributes, residual film and loose particles were poorly estimated by the PLSR and JRM models $($ Rcal $=0.12-0.32$, Table 2$)$. The lower Rcal values would be that these attributes were not directly related to the texture parameters from the texture analyzer. Residual feeling and loose particles (Table 1) were almost impossible to estimate by the texture analyzer. These characteristics were the perception by the mouth and tongue rather than the physical characteristics measured by the texture analyzer. Toothpack was moderately estimated by the PLSR $($ Rcal $=0.59)$ and the JRM $($ Rcal $=0.60)$. Since the definition of toothpack was 'the amount of product packed into the crowns of your teeth after mastication (Table 1)' it was related to the stickiness characteristic, which can be measured by the texture analyzer. The optimized model, 
however, did not show significant increase in the prediction of toothpack.

When looking at the attributes in each stage, the texture measurements in the initial, first bite/chew and chewdown stages could be estimated more accurately than the residual stage. Cohesion of bolus, adhesion to lips, and hardness showed strong Rcal based on the JRM (Rcal > 0.7). In addition, cohesiveness showed Rcal close to 0.7 . Texture sensory attributes for the chewdown stages showed moderate correlations with Rcal of 0.51 to 0.60 . The subjects evaluated the attributes in chewdown stage after several mastications; therefore, one-bite texture measurement system in this study might not reflect the attributes in chewdown stage accurately. Lee et al. (2009) also reported that such attributes being assessed throughout the chewdown stages as hardness of mass, cohesiveness of mass and number of chews were better predicted by the mastication assessment systems in poultry. The SSSA might have higher accuracy for estimating the texture sensory attributes at the early biting/chewing stage of cooked rice.

\section{CONCLUSION}

Two regression models - PLSR and JRM - were applied to estimate sensory texture attributes of cooked rice using the results from the texture analyzer. The JRM showed slightly stronger relations than the PLSR when estimating sensory attribute using the texture analyzer. Removing outliers from the full optimized model, PLSR, was proven to be effective to increase calibration model correlation coefficient and to decrease root mean square error of calibration in general. Due to the limitation of the texture analyzer, statistical models for estimating sensory texture attributes were appropriate only for sensory texture attributes directly related to the texture characteristics. Human evaluation is necessary for non-texture attributes of cooked rice. Throughout this study, cohesion of bolus, adhesion to lips, hardness, cohesiveness, toothpull and toothpack, which were directly related to the texture parameters, were able to estimate their intensities using the texture analyzer.

\section{Authors' contributions}

Study design and management: J. F. M. Conducted the experiments: M. L. Analysis and interpretation of data: M. L, H. S. K., Y. L. Drafting of manuscript: H. S. K. Critical revision: Y. L., J. F. M.

\section{REFERENCES}

Chen, J., C. Karlsson and M. Povey. 2005. Acoustic envelope detector for crispness assessment of biscuits. J. Texture. Stud. 36: 139-156.

Jeong, Y., Y. A. Jang, Y. H. Chang and Y. Lee. 2013. Prediction of consumer acceptability of potato chips with various moisture contents using survival analysis. Food Sci. Biotechnol. 22: 16211627.

Juliano, B. B., C. M. Perez, E. P. Alyo-Shing, V. B. Ramanov, A. B. Blakeney, L. A. Welsh, N. H. Choudhyry, L. L. Delgado, T. Iwasakl, N. Shibuya, A. P. Mossman, B. Siwi, D. S. Damardjati, H. Suzuki and H. Kimura. 1984. International cooperative test on texture of cooked rice. J. Texture Stud. 15: 357-376.

Kwak, H. S., M. Kim, Y. Lee and Y. Jeong. 2015a. Identification of key sensory attributes for consumer acceptance and instrumental quality of aseptic-packaged cooked rice. Int. J. Food Sci. Technol. 50: 691-699.

Kwak, H. S., M. Kim and Y. Jeong. 2015b. Physicochemical properties and determination of key instrumental quality measurement parameters of frozen-cooked rice by correlating consumer acceptance. J. Food Qual. 38: 192-200.

Lee, Y. S., C. M. Owens and J. F. Meullenet. 2008. A novel laser air puff and shape profile method for predicting tenderness of broiler breast meat. Poult. Sci. 87: 1451-1457.

Lee, Y. S., C. M. Owens and J. F. Meullenet. 2009. Tenderness perception of poultry major pectoralis muscle during mastication. J. Food Sci., 74: S413-S422.

Lee, Y., R. Xiong and J. F. Meullenet. 2014. Prediction of sensory texture of broiler breast meat using instrumental spectral stressstrain analysis. Int. J. Food Sci. Technol. 49: 2269-2275.

Lenzo, M. and J. F. Meullenet. 2000. Prediction of rice sensory texture attributes using spectral strain stress analysis and the jack-knife model optimization method. Discov. Mag. 1: 40-46.

Martens, H. and M. Martens. 2001. Multivariate analysis of quality. An Introduction. Wiley, Chichester, UK.

Meilgaard, M., G. V. Civille and B. T. Carr. 1999. Sensory Evaluation Techniques. 3rd ed. CRC Press, Inc., Boca Raton, Florida, USA.

Meullenet, J. F. C., J. Gross, B. P. Marks and M. Daniels. 1998. Sensory descriptive texture analyses of cooked rice and its correlation to instrumental parameters using an extrusion cell. Cereal Chem. 75: 714-720.

Meullenet, J. F. C., C. Sitakalin and B. P. Marks. 1999. Prediction of rice texture by spectral stress strain analysis: a novel technique for treating instrumental extrusion data used for predicting sensory texture profiles. J. Texture Stud. 30: 435-450.

Nishinari, K. 1996. New texture modifiers for foods, interactions among different food hydrocolloids and their potential of application. Kagaku Seibutsu. 34: 197-204.

Okabe, M. 1979. Texture measurement of cooked rice and its relationship to the eating quality. J. Texture Stud. 10: 131-152.

Sitakalin, C. and J. F. C. Meullenet. 2000. Prediction of cooked rice texture using extrusion and compression tests in conjunction with spectral stress strain analysis. Cereal Chem. 77: 501-506. 